\title{
Quadrados Mínimos e Modelo Misto no Acesso à Estimativa da Resposta Direta à Seleção em Rebanhos Experimentais de Bovinos Nelore
}

\author{
Maria Eugênia Zerlotti Mercadante1, 3, Irineu Umberto Packer2, 4, Alexander George Razook 1, 4, \\ Joslaine Noely dos Santos Gonçalves Cyrillo ${ }^{1,5}$
}

\begin{abstract}
RESUMO - Registros do peso à seleção de bovinos Nelore pertencentes aos rebanhos experimentais da Estação Experimental de Zootecnia de Sertãozinho, foram usados para estudar o acréscimo de informações no acesso à estimativa da mudança genética anual, utilizando métodos de quadrados mínimos e de modelo misto. A mudança genética nos três rebanhos (controle-NeC, e selecionados-NeS e $\mathrm{NeT}$ ) ou somente nos selecionados ( $\mathrm{NeS}$ e $\mathrm{NeT}$ ) foi estimada, só para fêmeas, por quadrados mínimos, por modelo misto considerando somente o desempenho das fêmeas e incorporando a matriz de parentesco, e por modelo misto incorporando todas as informações disponíveis (registros de machos e fêmeas e matriz de parentesco). A incorporação de todas as informações disponíveis em modelo misto para estimar a mudança genética ao longo dos anos forneceu uma curva mais suavizada das médias anuais e, consequentemente, uma estimativa da tendência genética anual com menor erro-padrão. O método dos quadrados mínimos forneceu estimativas da mudança genética ao longo dos anos altamente variáveis, evidenciando a menor capacidade de particionar a mudança genética da mudança ambiental. O modelo misto incorporando parte das informações de desempenho forneceu estimativas da tendência genética anual muito baixas (viesadas), evidenciando o confundimento da mudança ambiental com o restante da mudança genética não estimada pela ausência de informações.
\end{abstract}

Palavras-chave: experimento de seleção, mudança genética, tendência genética anual

\section{Least Squares and Mixed Model to Access the Estimate of Direct Response to Selection in Nelore Experimental Herds}

\begin{abstract}
Records of body weight at selection of Nelore cattle pertaining to the Sertãozinho's Experimental Station (SP-Brasil) Nelore herds were used to study the influence of the incorporation of additional information on the estimation of annual genetic trend by least squares and mixed model methodologies. The genetic changes in all three Nelore herds (control-NeC and selected-NeS and NeT) or only in the selected ones ( $\mathrm{NeS}$ and $\mathrm{NeT}$ ) were estimated, only for the females, by least squares, by mixed model including their performances and the relationship matrix, and by mixed model incorporating all available information (males and females records and the relationship matrix). The incorporation of all information provided a smoother curve of the annual means throughout the years and consequently an annual genetic trend estimate with lower standard error. Least squares provided highly variable annual estimates showing a lower capability of partitioning the genetic change from the environmental change. The mixed model analysis utilizing part of the performance records provided very low annual genetic trend estimates showing a confounding of the environmental with the genetic change.
\end{abstract}

Key Words: annual genetic trend, genetic change, selection experiment

\section{Introdução}

$\mathrm{Na}$ análise de experimentos de seleção com grandes animais, em que normalmente é impossível o controle das flutuações ambientais, uma complicação na estimação da resposta à seleção é com certeza a distinção entre as mudanças genética e ambiental (Hill, 1980; Falconer \& Mackay, 1996; Walsh, 1999).
Outro complicador é a variância considerável da resposta, em parte atribuída à deriva genética, uma vez que a seleção artificial envolve a escolha de pequeno número de pais para formar a próxima geração (Walsh, 1999). Falconer \& Mackay (1996) ressaltam ainda o problema da variabilidade das médias por geração (ou ano), as quais não avançam de maneira simples e regular, mas flutuam

\footnotetext{
${ }^{1}$ Estação Experimental de Zootecnia de Sertãozinho- IZ - C.P. 63 - Cep:14.160-900 - Sertãozinho-SP. E.mail: mezmerca@ig.com.br, iupacker@carpa.ciagri.usp.br; razook@iz.sp.gov.br; cyrillo@iz.sp.gov.br

2 Departamento de Produção Animal - ESALQ-USP - Piracicaba-SP.

${ }^{3}$ Bolsa de doutorado CNPq.

${ }^{4}$ Bolsa de produtividade do CNPq

5 Bolsa de doutorado da CAPES
} 
erraticamente e mais ou menos violentamente, e a consequência disso é que a resposta raramente pode ser medida com acurácia até passar, pelo menos, as duas primeiras gerações de seleção. O método mais comumente usado na análise de experimentos delineados é o de quadrados mínimos, enquanto o método usual de escolha na análise de dados de campo baseia-se na metodologia de modelos mistos, em virtude das suas propriedades estatísticas bem definidas (Kennedy, 1990; Meyer \& Hill, 1991).

Em um experimento, a resposta à seleção pode ser estimada usando procedimentos de quadrados mínimos, como a média das progênies dos pais selecionados e, neste caso, os efeitos ambientais são estimados pelo uso de uma população controle não selecionada (Frahm et al., 1985; Baker et al., 1991; Parnell et al., 1997; Razook et al., 1998). Em contraste com o estimador de modelo misto (considerando um modelo animal), o de quadrados mínimos usa somente os registros da geração i para calcular a média da geração i, sem usar a informação das outras gerações, ou seja, a análise de quadrados mínimos ignora a estrutura dos dados, usando somente os registros de uma geração em particular para computar a resposta e ponderar igualmente todos os indivíduos selecionados (Walsh, 1999).

Alternativamente, procedimentos de modelos mistos podem ser usados para analisar os dados, produzindo estimativas da variância genética e da resposta à seleção numa única análise (Sorensen \& Kennedy, 1986). A metodologia de modelos mistos para o modelo animal incorpora a matriz das relações genético-aditivas, levando em conta todas as covariâncias (tanto aquelas entre indivíduos dentro de uma geração como aquelas entre indivíduos de diferentes gerações), além de considerar o fato que algumas famílias fornecem mais informações e de ponderar corretamente os indivíduos dessas famílias usando registros da geração corrente e de todas as gerações passadas para estimar a resposta à seleção. A omissão do relacionamento entre gerações tem implicações importantes na análise de dados que foram gerados por seleção (Sorensen \& Kennedy, 1984, 1986), já que experimentos de seleção são processos estocásticos e o desempenho em determinada geração é dependente da amostra genética retida na geração anterior (Hill, 1980).

O objetivo deste trabalho foi ilustrar a incorporação do parentesco e de registros de desempenho no acesso à estimativa da mudança genética, utilizando registros do caracter sob seleção direta, peso ao sobreano, e procedimentos de quadrados mínimos e modelos mistos, em três rebanhos experimentais de bovinos Nelore.

\section{Material e Métodos}

As informações são provenientes do Experimento de Seleção da Estação Experimental de Zootecnia de Sertãozinho, iniciado em 1976. O estabelecimento dos rebanhos Nelore Controle $(\mathrm{NeC})$, Nelore Seleção $(\mathrm{NeS})$ e Nelore Tradicional $(\mathrm{NeT})$ ocorreu na estação de monta de 1980 , com respectivamente 60 , 120 e 170 fêmeas e quatro, seis e até oito touros/ano, efetivo que foi mantido ao longo dos anos. Os machos foram selecionados segundo o diferencial de seleção máximo ( $\mathrm{NeS}$ e $\mathrm{NeT})$ e nulo $(\mathrm{NeC})$, dentro de rebanho $\mathrm{x}$ ano, do peso padronizado aos 378 dias (P378), obtido após prova de desempenho em confinamento, e as fêmeas segundo o diferencial de seleção máximo ( $\mathrm{NeS}$ e $\mathrm{NeT}$ ) e nulo $(\mathrm{NeC})$ do peso padronizado aos 550 dias (P550), em regime de pastagem. O NeT, em um esquema de reposição de touros e matrizes mais flexível, recebeu no início touros de fora dos rebanhos da estação, assim como, eventualmente, touros e matrizes descartados dos $\mathrm{NeC}$ e NeS. Detalhes da implantação dos rebanhos e do processo seletivo foram descritos por Razook et al. (1998).

Os pesos à seleção dos machos (P378) e das fêmeas (P550) foram calculados somando ao peso de desmame, padronizado para 210 dias, o ganho de peso pós-desmame em confinamento até um ano para os machos, e o ganho de peso pós-desmame em regime de pastagem até um ano e meio para as fêmeas. Para as análises de quadrados mínimos foram utilizados registros das fêmeas (P550) nascidas de 1981 a 1998. Para a análise de P550 por modelo misto foram incorporados também os registros do P550 das fêmeas nascidas de 1978 a 1980, antes da separação dos rebanhos, identificados como rebanho original. $\mathrm{Na}$ análise de modelo misto utilizando todas as informações disponíveis (animais nascidos de 1978 a 1998), os pesos de machos e fêmeas, ou seja, o peso aos 378 dias de idade (P378) e o peso aos 550 dias de idade (P550) respectivamente, foram analisados como um só caracter (PSEL), padronizando-os ou não pelo respectivo desvio-padrão de cada sexo $(35,78$ e $39,20 \mathrm{~kg}$ para machos e fêmeas, respectivamente), como sugerido por Koch et al. (1994). A matriz de parentesco completa com 5830 animais, sendo os animais base

R. Bras. Zootec., v.32, n.5, p.1123-1130, 2003 
(410) os pais dos touros e matrizes usados de 1978 a 1980, foi incorporada nas análises de modelo misto, tanto de P550 como de PSEL. A estrutura geral dos dados utilizados é mostrada na Tabela 1 .

As médias de quadrados mínimos para P550, dentro de rebanho-ano de nascimento, foram obtidas no Proc Mixed do SAS (2000), com modelo ajustando os efeitos fixos de rebanho ( $\mathrm{NeC}, \mathrm{NeS}$ e $\mathrm{NeT}$ ), ano de nascimento $(1981, \ldots, 1998)$, mês de nascimento $(8, \ldots, 11)$ e idade da mãe em anos $(3, \ldots, 10)$, além da interação ano x rebanho. Os componentes de variância foram estimados por máxima verossimilhança restrita (REML) usando o software ASREML (Gilmour et al., 2000). Para P550 o modelo misto incluiu os efeitos fixos descritos acima, além dos efeitos aleatórios genético direto do animal e permanente da mãe. $O$ modelo para PSEL foi o mesmo daquele ajustado para P550, além do efeito fixo de sexo e as interações rebanho $\mathrm{x}$ sexo e ano $\mathrm{x}$ sexo, uma vez que a análise de PSEL considera P378 (machos) e P550 (fêmeas) como um só caracter. Análises preliminares, testando diferentes modelos (por meio do teste da razão de verossimilhança), indicaram ser este o melhor modelo para P550 e PSEL, em vez do "modelo completo" (contendo efeitos genéticos aditivo direto e materno e efeito permanente de ambiente).

Como na análise de modelos mistos os valores genéticos são dependentes da estimativa de herdabilidade na população base, eles serão subestimados se a herdabilidade for menor que o verdadeiro valor, e serão superestimados se a herdabilidade for maior (Sorensen \& Kennedy, 1984). Entretanto, a

Tabela 1 - Estrutura dos dados

Table 1 - Data structure

\begin{tabular}{|c|c|c|}
\hline $\begin{array}{l}\text { Item } \\
\text { Item }\end{array}$ & $\mathrm{P}^{250^{1}}$ & PSEL $^{2}$ \\
\hline $\begin{array}{l}\text { Animais com registro } \\
\text { Animals with record }\end{array}$ & 2.353 & 4.677 \\
\hline $\begin{array}{l}\text { Touros } \\
\text { Sires }\end{array}$ & 161 & 166 \\
\hline $\begin{array}{l}\text { Vacas } \\
\text { Dams }\end{array}$ & 953 & 1160 \\
\hline $\begin{array}{l}\text { Média }(\mathrm{kg}) \\
\text { Mean }\end{array}$ & 263 & 282 \\
\hline $\begin{array}{l}\text { Desvio-padrão }(\mathrm{kg}) \\
\text { Standard deviation } \\
\end{array}$ & 39 & 42 \\
\hline \multicolumn{3}{|c|}{$\begin{array}{l}1 \text { P550: peso padronizado aos } 550 \text { dias de idade (só fêmeas). } \\
2 \text { PSEL: peso à seleção, pesos padronizados aos } 378 \text { dias de } \\
\text { idade (só machos) e aos } 550 \text { dias de idade (só fêmeas). } \\
1 \text { P550: corrected weight at } 550 \text { days of age (only females). } \\
2 \text { PSEL: selection weight, corrected weights at } 378 \text { days of age (only males) } \\
\text { and at } 550 \text { days of age (only females). }\end{array}$} \\
\hline
\end{tabular}

R. Bras. Zootec., v.32, n.5, p.1123-1130, 2003 herdabilidade na população base não é conhecida, e no presente estudo os valores genéticos foram estimados para P550 e PSEL com a herdabilidade estimada diretamente dos dados. Neste tipo de análise, uma suposição importante para que os componentes de variância e, portanto, a herdabilidade e os valores genéticos estimados não sejam viesados em conseqüência da seleção é que, com seleção direcional, as mudanças nas freqüências alélicas são infinitamente pequenas e a mudança na variância genética é conseqüencia quase que exclusivamente da geração de desequilíbrio gamético entre os locos que afetam o carácter. Com número finito de locos, particularmente com alelos de grande efeito, esta suposição é menos sustentável e, sem dúvida, o ponto mais crítico para o uso de modelo misto na análise de experimentos de seleção (Sorensen \& Kennedy, 1984, 1986; Kennedy, 1990; Meyer \& Hill, 1991).

Pelo método dos quadrados mínimos, a resposta genética anual nos rebanhos selecionados $\mathrm{NeS}$ e $\mathrm{NeT}$ foi estimada como o desvio das médias anuais ajustadas em relação ao $\mathrm{NeC}$, como sugerido por Frahm et al. (1985), Baker et al. (1991), Parnell et al. (1997) e Razook et al. (1998), e a tendência genética como a regressão dos desvios em função do ano de nascimento (Falconer \& Mackay, 1996). Uma vez que os animais dos três rebanhos foram manejados de maneira similar, qualquer diferença observada no desempenho médio dos rebanhos pode ser atribuída à resposta genética à seleção (Parnell et al., 1997). Pelo metodologia de modelos mistos, a estimativa da resposta genética foi obtida pela média dos valores genéticos diretos de P550 e PSEL para cada ano de nascimento e para cada um dos rebanhos, e a tendência genética, por meio da regressão dessas médias em função do ano de nascimento do animal. Para PSEL este procedimento foi feito somente para as fêmeas, embora as soluções tenham sido obtidas em análise com machos e fêmeas. A tendência ambiental, por modelo misto, foi obtida da regressão das soluções de ano de nascimento para P550 e de ano de nascimento x sexo (fêmea) para PSEL, em função do ano de nascimento.

\section{Resultados e Discussão}

As médias ajustadas de P550 para os três rebanhos, por ano de nascimento, fornecem por si uma idéia da mudança fenotípica ocorrida ao longo dos anos (Figura 1). Observa-se grande variabilidade entre ano nas médias fenotípicas para cada rebanho, 
mas com tendência de paralelismo para todos os rebanhos, sugerindo efeitos ambientais não identificados intrínsecos a cada ano. Ainda, segundo Walsh (1999), esta variância considerável da resposta pode também, em parte, ser atribuída à deriva genética, uma vez que a seleção anual envolve a escolha de pequeno número de pais, principalmente de touros, para formar a próxima geração. Há tendência mais clara de diferenciação das médias anuais dos rebanhos selecionados ( $\mathrm{NeS}$ e $\mathrm{NeT}$ ) e do controle somente após o ano de 1990, o que concorda com a observação feita por Falconer \& Mackay (1996), que a resposta somente pode ser medida com certa acurácia após as duas primeiras gerações de seleção.

A mudança genética em P550 no decorrer do experimento de seleção, obtida por quadrados mínimos como o desvio das médias ajustadas de $\mathrm{NeS}$ e $\mathrm{NeT}$ em relação ao controle, está ilustrada na Figura 2. Observa-se que, mesmo após a correção para as mudanças ambientais (desvio da população controle), as médias anuais continuam a flutuar de modo extremamente variável. Permanecem ainda a influência de fatores ambientais não identificáveis e a variância devido à deriva genética. Principalmente por razões econômicas, em bovinos, geralmente a população controle possível de ser estabelecida e mantida é pequena, agravando o problema da deriva aleatória, resultando em maior erro na estimação da verdadeira resposta à seleção (Walsh, 1999). Segundo Hill (1980), assegurando um diferencial de seleção

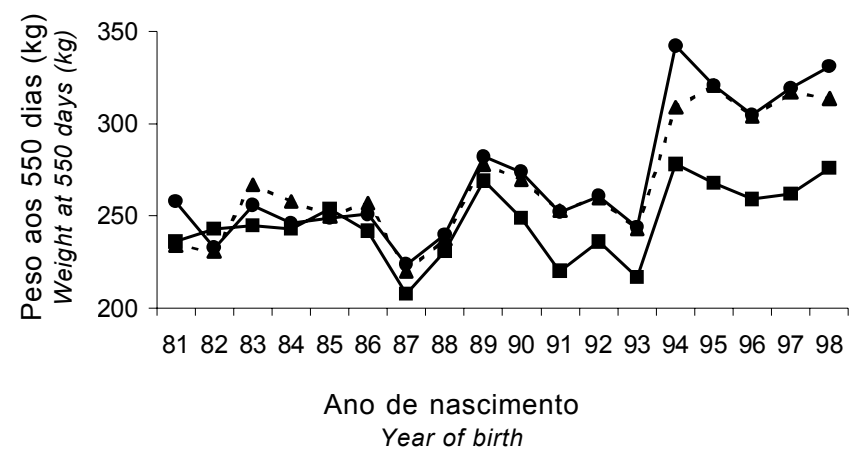

Figura 1 - Médias anuais ajustadas do peso aos 550 dias de idade (P550) das fêmeas dos três rebanhos (controle $\mathbf{\square}$, seleção $\bullet$, tradicional $\mathbf{\Delta}$ ), representando a mudança fenotípica, estimada por quadrados mínimos.

Figure 1 - Adjusted annual means of the weight at 550 days of age (P550) of females from the three herds (control $\mathbf{\square}$, selection •, traditional $\mathbf{\Delta ) , ~ r e p r e s e n t i n g ~ t h e ~}$ phenotypic changes, estimated by least squares. de zero ou próximo de zero, como no caso do presente experimento, a variância devido à deriva, na população controle, pode ser minimizada.

Hill (1980) e Sorensen \& Kennedy $(1984,1986)$ alertam que o desempenho em uma dada geração é dependente da amostra genética retida na geração anterior e, assim, a omissão do relacionamento entre gerações pode ter implicações importantes na análise de dados que foram gerados por seleção. A incorporação das relações entre todos os animais, dentro e entre as gerações, contorna os problemas que contribuem para a variância da resposta. Na Figura 3, as médias anuais dos valores genéticos para P550, obtidas em análise de modelo misto sob modelo animal, incorporando todo o parentesco, ilustram a menor flutuação anual quando comparadas às médias de quadrados mínimos mostradas nas figuras anteriores, já que o relacionamento entre as gerações está sendo considerado nessa análise. Entretanto, aqui ainda observa-se a mesma tendência de maior variabilidade até passar cerca de duas gerações de seleção, momento em que as médias de P550 dos rebanhos selecionados ( $\mathrm{NeS}$ e $\mathrm{NeT}$ ) distanciam-se consistentemente daquela do controle.

$\mathrm{Na}$ análise da mudança genética de P550 por modelo misto, apesar da incorporação de todo o relacionamento de parentesco entre os animais desde a implantação do experimento, a avaliação genética do peso das fêmeas só está em função do próprio desempenho e do desempenho dos parentes fêmeas,

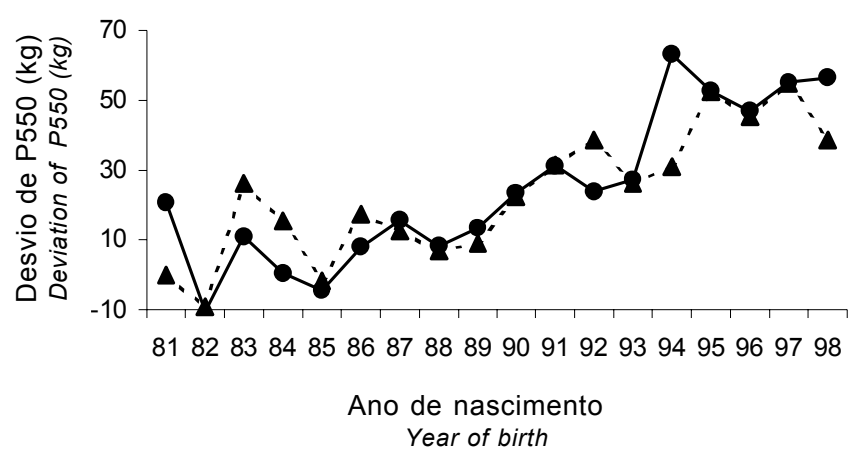

Figura 2 - Desvios do peso aos 550 dias de idade (P550) das fêmeas dos rebanhos $\mathrm{NeS}$ e $\mathrm{NeT}$ (seleção •, tradicional ^), em relação à média ajustada de $\mathrm{NeC}$, representando a mudança genética, estimada por quadrados mínimos.

Figure 2 - Deviation of the weight at 550 days of age (P550) of females of the NeS and NeT herds (selection •, traditional \) from $\mathrm{NeC}$ adjusted means, representing the genetic change, estimated by least squares.

R. Bras. Zootec., v.32, n.5, p.1123-1130, 2003 
uma vez que o registro de desempenho dos machos, pelo fato de ter sido obtido em idade e manejos pósdesmama distintos, não foi considerado. Na análise por modelo misto incorporando toda a informação disponível, os registros de desempenho à seleção de machos (P378) e fêmeas (P550) foram analisados como um só (PSEL) em modelo animal, padronizando-os ou não pelo respectivo desvio-padrão de cada sexo (35,78 kg para machos e 39,20 kg para fêmeas), como sugerido por Koch et al. (1994). As soluções obtidas por ambos procedimentos foram muito similares, e as médias anuais dos valores genéticos de PSEL para cada rebanho, obtidas na análise sem padronização, são mostrados na Figura 4.

Juntamente com a Figura 4, é possível observar que conforme as informações da população estudada vão sendo incorporadas na análise (em ordem crescente, Figura 2, sem parentesco e sem registros dos machos e Figura 3, com parentesco e sem registros dos machos), menores são as flutuações anuais. $\mathrm{O}$ uso da matriz de parentesco tem o efeito de comparar o registro do animal com um índice baseado nas informações de seus parentes, e os valores genéticos individuais são regredidos em direção ao valores preditos pelo índice, suavizando flutuações excessivas (Walsh, 1999). Em virtude desta propriedade, a resposta estimada por modelo misto tende a ser mais suave que a resposta verdadeira (ou aquela estimada por quadrados mínimos), pois é menos afetada pelos

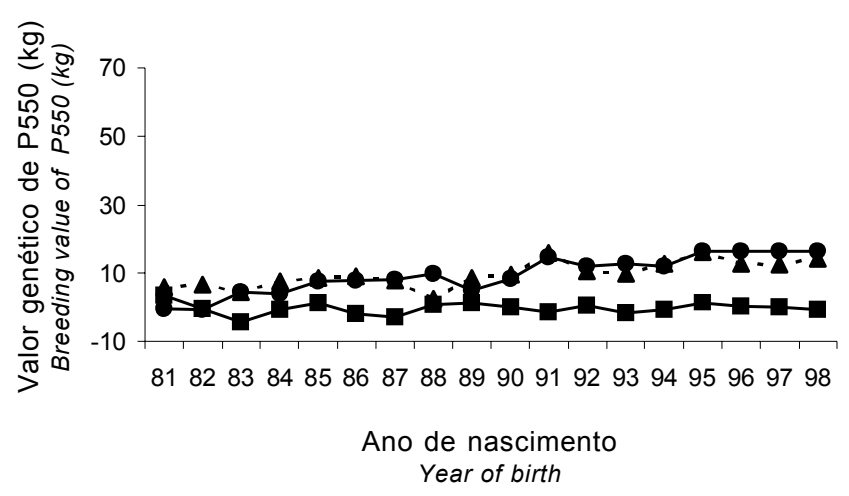

Figura 3 - Médias anuais dos valores genéticos do peso aos 550 dias de idade (P550) das fêmeas dos três rebanhos (controle $\mathbf{a}$, seleção •, tradicional $\Delta)$, representando a mudança genética estimada por modelo misto, sem incluir registro dos machos.

Figure 3 - Annual breeding values means of weight at 550 days of age (P550) of females from three herds (control $\mathbf{\square}$, selection $\bullet$, traditional $\mathbf{\Delta}$ ), representing the genetic change, estimated by mixed model, without record of males. verdadeiros desvios de amostragem (Sorensen \& Kennedy, 1986). Observa-se ainda, na Figura 4, logo nos primeiros anos do experimento, a nítida separação dos rebanhos selecionados ( $\mathrm{NeS}$ e $\mathrm{NeT})$ e controle, e não somente após as duas gerações de seleção como observado nas figuras anteriores.

Entretanto, sob modelo animal, os valores genéticos são expressos como desvios da média de todos os animais da população base (anterior a 1978) e, portanto, é imprescindível a definição correta da população base, especialmente em experimentos de seleção. Com a matriz de parentesco, animais sem pais identificados são tratados como animais da população base com a suposição de que foram amostrados de uma única população com média do valor genético nula e de mesma variância genética aditiva (Kennedy et al., 1988). Tal deficiência pode ser superada considerando modelo que leve em conta a estrutura de subpopulação, como o uso de grupos genéticos para novos animais sem pais conhecidos que entram na população no decorrer do tempo.

Apesar da diferença de comportamento das médias anuais mostradas nas três figuras anteriores (Figuras 2, 3 e 4), observa-se em todas elas, com menor ou maior evidência, dois fatos que realmente ocorreram nos rebanhos: o uso de touros com diferenciais de seleção positivo em NeS no estabelecimento do experimento (1978-1980) mostrado pelo maior valor para $\mathrm{NeS}$ na primeira progênie (1981) em

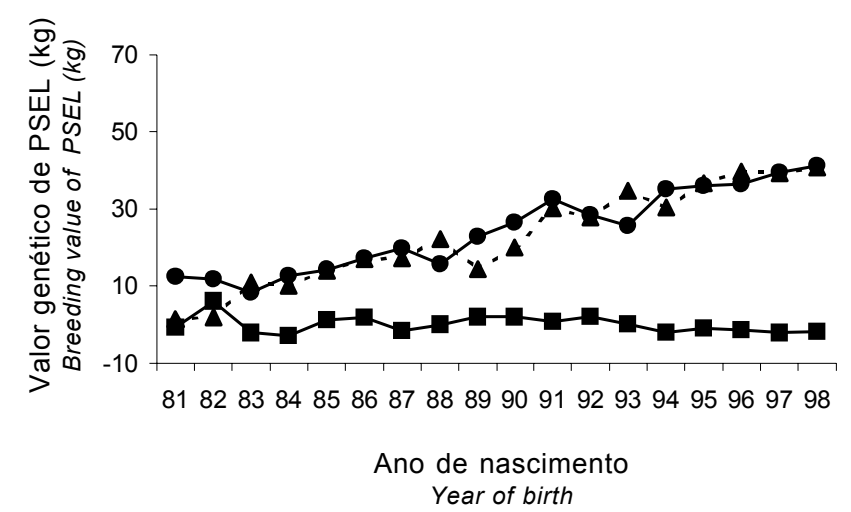

Figura 4 - Médias anuais dos valores genéticos do peso à seleção (PSEL) das fêmeas dos três reba-

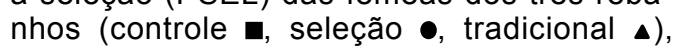
representando a mudança genética estimada por modelo misto, incluindo todas as informações disponíveis.

Figure 4 - Annual breeding values means of selection weight (PSEL) of females from three herds (control $\mathbf{n}$, selection $\bullet$, traditional $\Delta$ ), representing the genetic change, estimated by mixed model, including all information available.

R. Bras. Zootec., v.32, n.5, p.1123-1130, 2003 
relação a $\mathrm{NeC}$ e $\mathrm{NeT}$; e o uso de dois touros provenientes do NeC no rebanho NeT na monta de 1988, fato que se refletiu na diminuição do valor anual em 1989. Como esperado, estes fatos puderam ser melhor visualizados na Figura 4. A mudança ambiental, obtida das soluções de ano de nascimento da análise de PSEL, é mostrada na Figura 5. As flutuações da Figura 5 acompanham quase que fielmente as flutuações das médias anuais da Figura 1.

As tendências genéticas anuais para as fêmeas em 18 anos de seleção, estimadas pelo regressor das médias, obtidas pelos diferentes procedimentos, em função do ano de nascimento, estão na Tabela 2. A tendência fenotípica estimada no rebanho controle é o estimador da mudança ambiental pelo método de quadrados mínimos. A tendência genética por modelo misto e considerando somente registros de fêmeas (P550) parece estimar somente parte da tendência genética conseguida nos rebanhos selecionados (NeS e $\mathrm{NeT}$ ) e, consequentemente, sua correspondente tendência ambiental pode estar inflacionada pela porção da tendência genética não considerada (registros dos machos). Pelo modelo misto, considerando todas as informações disponíveis (PSEL), as tendências genética e ambiental, ao menos teoricamente, foram estimadas levando-se em conta os acasalamentos não aleatórios (seleção) e, portanto, podem ser vistas como as menos viesadas, ou as "verdadeiras". Como esperado, a tendência genética estimada para cada um dos rebanhos por modelo misto, incorporando todas as informações disponíveis apresentou, relativamente, o menor erro-padrão.

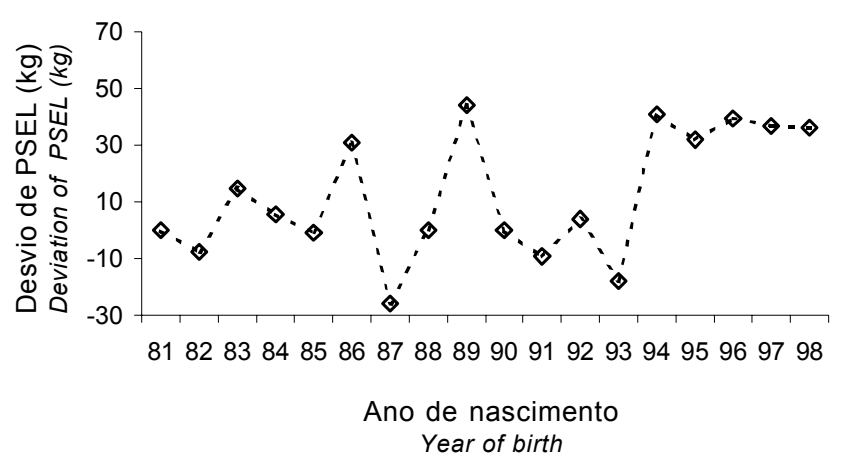

Figura 5 - Mudança ambiental do peso à seleção (PSEL) das fêmeas, apresentada como desvio de 1981.

Figure 5 - Environmental change of selection weight (PSEL) of females, shown as the deviation of 1981 .
Assim, apesar de a metodologia de modelos mistos permitir estimar tendências genéticas sem a necessidade de uma população controle, suas estimativas ficam muito comprometidas quando uma parte significativa dos dados que orientaram a seleção não é utilizada (neste caso, os dados das provas de ganho de peso dos machos), e, portanto, a análise de P550, por modelo misto, mas omitindo as informações do desempenho dos machos parece ser o modelo menos correto para estimar mudança e tendência genética. Em outra análise, para P378 de machos, com modelo misto, omitindo as informações do desempenho das fêmeas, os resultados mostraram a mesma tendência de subestimação (viés) nas estimativas da tendência genética, como no caso de P550 das fêmeas, omitindo o desempenho dos machos. Para os rebanhos NeS e $\mathrm{NeT}$ os valores de tendência genética para P378 foram: $1,18 \pm 0,10 \mathrm{~kg} /$ ano e $1,43 \pm 0,11 \mathrm{~kg} /$ ano, respectivamente, e foram mais baixos quando comparados aos resultados obtidos para os machos na análise de PSEL, quando todas as informações disponíveis foram incluídas, $(1,69 \pm 0,13 \mathrm{~kg} /$ ano para $\mathrm{NeS}$ e 2,26 $\pm 0,15 \mathrm{~kg} /$ ano para $\mathrm{NeT}$ ), resultados não mostrados na Tabela 2.

Os coeficientes de determinação $\left(\mathrm{R}^{2}\right)$ dos modelos de regressão para a obtenção das tendências genéticas e ambientais refletem o que já foi discutido anteriormente. Cerca de $93 \%$ da variação das médias anuais dos valores genéticos de PSEL obtidos em modelo misto foi explicada pelo ano de nascimento, ou seja, pela seleção direta para peso que foi sendo acumulada no decorrer dos anos. Para os desvios em relação ao controle, obtidos por quadrados mínimos, o ano de nascimento explicou 75 e $71 \%$, da variação em NeS e NeT, e o valor mais baixo de $\mathrm{R}^{2}$ nos rebanhos selecionados foi obtido no modelo animal com P550 ignorando as informações do desempenho dos machos $(60 \%$ para $\mathrm{NeS})$. Para o $\mathrm{NeC}$, todos os métodos forneceram $\mathrm{R}^{2}$ próximos de zero, indicando não haver relação das variações anuais em função do ano de nascimento, resultado esperado para um rebanho selecionado considerando-se diferenciais de seleção nulos.

$\mathrm{Na}$ apresentação e discussão de métodos para estimar a mudança genética em experimentos de seleção, cabe ainda uma consideração sobre a necessidade de manutenção de uma população controle. Segundo Blair \& Pollak (1984), vários pontos merecem atenção: 1) na abordagem de modelo misto, para separar, com sucesso, mudanças genética e ambiental, são necessários fortes laços genéticos entre os anos e se

R. Bras. Zootec., v.32, n.5, p.1123-1130, 2003 
Tabela 2 - Estimativas das tendências fenotípica, genética e ambiental anuais para as fêmeas, e respectivos coeficientes de determinação $\left(R^{2}\right)$ da equação de regressão

Table 2 - Estimates of phenotypic, genetic and environmental annual trends for females, and corresponding coefficients of determination of the regression equation

\begin{tabular}{|c|c|c|c|c|c|}
\hline \multirow[t]{2}{*}{$\begin{array}{l}\text { Tendência (kg/ano) } \\
\text { Trend (kg/year) }\end{array}$} & \multirow[t]{2}{*}{$\begin{array}{l}\text { Método } \\
\text { Method }\end{array}$} & \multirow[t]{2}{*}{$\begin{array}{c}\text { Caracter }^{2} \\
\text { Trait }\end{array}$} & \multicolumn{3}{|c|}{$\begin{array}{c}\text { Rebanho }^{3} \\
\text { Herd }\end{array}$} \\
\hline & & & $\mathrm{NeC}$ & $\mathrm{NeS}$ & $\mathrm{NeT}$ \\
\hline Fenotípica & $\mathrm{QM}(L S)$ & P550 & $1,59 \pm 0,84+$ & $5,19 \pm 1,10 * *$ & $4,44 \pm 1,21 * *$ \\
\hline Phenotypic & & & $(0,18)$ & $(0,44)$ & $(0,42)$ \\
\hline Genética & $\mathrm{QM}(L S)$ & P550 & & $3,60 \pm 0,52 * *$ & $2,93 \pm 0,46^{* *}$ \\
\hline Genetic & & & & $(0,75)$ & $(0,71)$ \\
\hline $\begin{array}{l}\text { Ambiental } \\
\text { Environmental }\end{array}$ & $\mathrm{QM}(L S)$ & P550 & $\begin{array}{c}1,59 \pm 0,84+ \\
(0,18)\end{array}$ & & \\
\hline Genética & $\mathrm{MM}(M M)$ & P550 & $0,02 \pm 0,08$ & $0,55 \pm 0,11 * *$ & $0,97 \pm 0,09 * *$ \\
\hline Genetic & & & $(0,00)$ & $(0,60)$ & $(0,87)$ \\
\hline Ambiental $^{4}$ & $\mathrm{MM}(M M)$ & P550 & $3,54 \pm 0,98 * *$ & $3,54 \pm 0,98 * *$ & $3,54 \pm 0,98 * *$ \\
\hline Environmental & & & $(0,45)$ & $(0,45)$ & $(0,45)$ \\
\hline Genética & $\mathrm{MM}(M M)$ & PSEL & $-0,13 \pm 0,10$ & $1,92 \pm 0,13 * *$ & $2,32 \pm 0,14^{* *}$ \\
\hline Genetic & & & $(0,09)$ & $(0,93)$ & $(0,94)$ \\
\hline Ambiental $^{4}$ & $\mathrm{MM}(M M)$ & PSEL & $2,07 \pm 0,91 *$ & $2,07 \pm 0,91 *$ & $2,07 \pm 0,91^{*}$ \\
\hline Environmental & & & $(0,24)$ & $(0,24)$ & $(0,24)$ \\
\hline
\end{tabular}

${ }_{1} \mathrm{QM}$ : quadrados mínimos; MM: modelo misto (LS: least square; MM: mixed model).

2 P550: peso padronizado aos 550 dias de idade (só fêmeas); PSEL: peso à seleção, pesos padronizados aos 378 dias de idade (só machos) e aos 550 dias de idade (só fêmeas) (P550: corrected weight at 550 days of age (only females); PSEL: selection weight, corrected weights at 378 days of age [only males] and at 550 days of age [only females]).

${ }^{3} \mathrm{NeC}$ : Nelore controle, NeS: Nelore seleção, NeT: Nelore tradicional (NeC: Nelore control, NeS: Nelore selection, NeT: Nelore traditional.

${ }^{4}$ Obtida das soluções de ano de nascimento (obtained from the birth year solutions).

$+P<0,10 ;{ }^{*} P<0,05 ;{ }^{* *} P<0,01$

esses laços não estão garantidos no início do experimento, um grupo controle poderia ser incluído; 2) um grupo controle é freqüentemente incluído para permitir comparações fisiológicas entre os grupos selecionado e controle após várias gerações de seleção ter ocorrido; 3) os dados do grupo controle podem ser usados para calcular as herdabilidades estimadas necessárias para uma análise de modelos mistos; e 4) dados do grupo controle fornecerão estimativas dos efeitos fixos que podem ser comparadas com estimativas similares do grupo selecionado, permitindo estudo interessante da interação genótipo e ambiente. Ainda, se a linha selecionada é geneticamente relacionada à controle, o uso de todos os dados numa única análise pode fornecer estimativas dos efeitos fixos e aleatórios com variância mínima (Sorensen \& Kennedy, 1984).

\section{Conclusões}

O método dos quadrados mínimos forneceu estimativas da mudança genética ao longo dos anos altamente variáveis, evidenciando a menor capacidade de particionar mudança genética da mudança ambiental e, conseqüentemente, estimativa com erropadrão maior que a obtida por modelo misto.

O modelo misto incorporando parte das informa- ções de desempenho e omitindo parte essencial dos dados que orientou a seleção dos machos, forneceu estimativa da tendência genética anual muito baixa, evidenciando o confundimento da mudança ambiental com o restante da mudança genética não estimada pela ausência de informações.

A incorporação de todas as informações disponíveis em modelo misto para estimar a mudança genética ao longo dos anos forneceu uma curva mais suavizada das médias anuais e, conseqüentemente, estimativa da tendência genética anual com menor erro-padrão.

\section{Literatura Citada}

BAKER, R.L.; MORRIS, C.A.; JOHNSON, D.L. et al. Results of selection for yearling or 18-month weight in Angus and Hereford cattle. Livestock Production Science, v.29, p.277-296, 1991.

BLAIR, H.T.; POLLAK, E.J. Estimation of genetic trend in a seleted population with and without the use of a control population. Journal of Animal Science, v.58, p.878-886, 1984.

FALCONER, D.S.; MACKAY, T.F.C. Introduction to quantitative genetics. Essex: Longman, 1996. 464p.

FRAHM, R.R.; NICHOLS, C.G.; BUCHANAN, D.S. Selection for increased weaning or yearling weight in Hereford cattle. II. Direct and correlated responses. Journal of Animal Science, v.60, p.1385-1395, 1985.

R. Bras. Zootec., v.32, n.5, p.1123-1130, 2003 
GILMOUR, A.R.; CULLIS, B.R.; WELHAM, S.J. et al. ASREML reference manual. ftp://ftp.res.bbsrc.ac.uk/ pub/aar/. 2000.

HILL, W.G. Design of quantitative genetic selection experiments. In: ROBERTSON, A. (Ed.) Selection experiments in laboratory and domestic animals. Slough: CAB, 1980. p.1-13.

KOCH, R.M.; CUNDIFF, L.V.; GREGORY, K.E. Cumulative selection and genetic change for weaning or yearling weight or for yearling weight plus muscle score in Hereford cattle. Journal of Animal Science, v.72, p.864-885, 1994.

KENNEDY, B.W.; SCHAEFFER, L.R.; SORENSEN, D.A. Genetic properties of animal models. Journal of Dairy Science, v.71, p.17-26, 1988. Supplement.

KENNEDY, B.W. Use of mixed model methodology in analysis of designed experiments. In: GIANOLA, D.; HAMMOND, K. (Eds.) Statistical methods for genetic improvement of livestock. Berlin: Spring Verlag, 1990. p.77-97.

MEYER, K.; HILL, W.G. Mixed model analysis of a selection experiment for food intake in mice. Genetic Research, v.57, p.71-81, 1991.

PARNELL, P.F.; ARTHUR, P.F.; BARLOW, R. Direct response to divergent selection for yearling growth rate in Angus cattle. Livestock Production Science, v.49, p.297304, 1997.
RAZOOK, A.G.; FIGUEIREDO, L.A.; BONILHA NETO, L.M. et al. Selection for yearling weight in Nelore and Guzera zebu breeds: selection applied and response in 15 years of progeny. (compact disc). In: WORLD CONGRESS ON GENETICS APPLIED TO LIVESTOCK PRODUCTION, 6., 1998, Armidale. Proceedings. Armidale, 1998. CD-ROM. Session: Beef Cattle.

SAS INSTITUTE. SAS/STAT 2000: version 8. Cary, 2000.

SORENSEN, D.A.; KENNEDY, B.W. Estimation of response to selection using least-squares and mixed model methodology. Journal of Animal Science, v.58, p.1097-1106, 1984.

SORENSEN, D.A.; KENNEDY, B.W. Analysis of selection experiments using mixed model methodology. Journal of Animal Science, v.63, p.245-258, 1986.

WALSH, J.B. Mixed-model and bayesian analysis of shortterm selection experiments. http://biosci.arizona.edu/ zbook/volume_2/chapters/vol2_07.html. 1999.

Recebido em: 04/04/02

Aceito em: 17/02/03 